\title{
HBV preS mutations promote hepatocarcinogenesis by inducing endoplasmic reticulum stress and up-regulating inflammatory signaling
}

Guangwen Cao ( $\nabla$ gcao@smmu.edu.cn )

Second Military Medical University https://orcid.org/0000-0002-8094-1278

Wenbin Liu

Rui Pu

Shiliang Cai

Zixiong Li

Donghong Liu

Xinyu Zhou

Jianhua Yin

Xi Chen

Liping Chen

Jianfeng Wu

Xiaojie Tan

Xin Wang

\section{Article}

Keywords: Hepatitis B virus, preS mutation, carcinogenesis, inflammation, STAT3

Posted Date: January 24th, 2022

DOI: https://doi.org/10.21203/rs.3.rs-1231704/v1

License: @ (i) This work is licensed under a Creative Commons Attribution 4.0 International License. Read Full License 


\section{Abstract}

We aimed to elucidate the effects and underlying mechanisms of hepatitis B virus (HBV) preS mutations on hepatocarcinogenesis. The association between preS mutations and hepatocellular carcinoma (HCC) occurrence was evaluated using a cohort of 2114 HBV-infected patients. Combo mutations G2950A/G2951A/A2962G/C2964A and C3116T/T31C significantly increased HCC risk in patients without antiviral treatment, whereas preS2 deletion significantly increased the HCC risk in patients who received antiviral treatment. The preS1/preS2/S mutants and their wild-type counterpart were delivered into Sleeping Beauty $(S B)$ mouse models. preS1/preS2/S mutants induced a higher tumor rate and higher serum levels of inflammatory cytokines than did wild type-preS1/preS2/S in $S B$ mice. The preS1/pres2/S mutants induced dramatic alteration of gene expression profiles partially through inducing prooncogenic inflammatory cytokine. Through enhancing the retention of HBV S protein in endoplasmic reticulum (ER), the preS1/preS2/S mutants enhanced ER stress, induced the alteration in metabolism, affected the response to hypoxia, and upregulated the protein level of signal transducer and activator of transcription 3 (STAT3). Inhibiting the STAT3 pathway attenuated the positive effects of preS1/preS2/S mutants on cell proliferation. Thus, G2950A/G2951A/A2962G/C2964A, C3116T/T31C, and preS2 deletion promote hepatocarcinogenesis via inducing ER stress, metabolism, and pro-oncogenic STAT3 inflammatory pathways, which can be transformed into specific prophylaxis of HCC.

\section{Introduction}

Hepatocellular carcinoma (HCC) is the main histotype of primary liver cancer and the third leading cause of cancerrelated death worldwide.[1] Chronic Hepatitis B virus (HBV) infection contributes to $44-55 \%$ of global HCC.[2] Compared with other HCC-related etiological factors, chronic infection of HBV is associated with a 10-year earlier onset of HCC.[3] During hepatocarcinogenesis, HBV evolves gradually, which is partially induced by cytidine deaminases.[4-6]. HBV genotypes B and C, the two genotypes endemic in China, have distinct mutation patterns, especially in the preS region of HBV genome [7]. HBV preS and S gene encode three forms of surface proteins: large (preS1/preS2/S), middle (preS2/S), and small (S) surface antigen. These HBV envelope proteins are synthesized in endoplasmic reticulum (ER). Some HBV mutations, especially those accumulated in the preS gene (preS1, nt.2848-nt.3204; preS2, nt.3205-nt.154), are significantly associated with increased risks of HCC occurrence and recurrence.[8-12] Among these HCC-related pres mutations, G2950A, G2951A, A2962G, and C2964A are exclusively in genotype C HBV, and HBV preS deletion is mostly occurring in HBV genotype C.[8, 9]

The mechanisms by which the preS mutations improve hepatocarcinogenesis remain poorly understood. Current studies mainly focus on preS deletion, especially the preS2 deletion, rather than single or combo nucleotide substitutions within the preS region. The preS deletion-induced mutant envelope proteins accumulate in ER, leading to impaired secretion of HBsAg and ER stress. Experimental data from transgenic mice and HCC cells demonstrate that HBV preS deletion activates carcinogenic signaling pathways in an ER stress-dependent way.[13-15] The preS deletion induced ER stress also leads to calcium overload in mitochondria, reduction of ATP production, and liver fibrosis, thereby promoting HCC.[16] However, the effects and underlying mechanisms of combo pres mutations on hepatocarcinogenesis are rarely investigated. Antiviral treatments with nucleos(t)ide analog (NA) and/or interferon a (IFNa) can reduce the risks of HCC occurrence and recurrence, especially in those with HBV mutations in core promoter region of the HBV genome.[17,18] However, the prophylactic effect of antiviral treatment on HCC in those with the preS mutations remains unknown. In this study, we evaluated the effects of the preS deletion and combo preS mutations on the occurrence of HCC in a cohort of HBV-infected patients. The oncogenic effects of preS mutations were then validated by using Sleeping Beauty $(S B)$ transposon system to deliver preS1/preS2/S mutants into the livers of fumarylacetoacetate hydrolase (Fah)-deficient mice. Molecules regulated by the preS mutations were identified through

Page 2/23 
gene expression profiling analysis. This study helps elucidate the mechanisms by which the preS mutants promote carcinogenesis and reveals potential therapeutic options for HBV-related HCC.

\section{Materials And Methods}

\section{Study population}

In total, 2114 HBV-infected patients were enrolled from the second affiliated hospital of Second Military Medical University. The inclusion criteria, exclusion criteria, and the diagnostic criteria of chronic HBV infection, liver cirrhosis, and HCC, were the same as previously described.[18] The information including antiviral treatments is detailed in the Supplementary Materials. Participants received regular follow-up examinations as previously described.[17] The last date of follow-up was 31 August 2019. The study protocol conformed to the 1975 Declaration of Helsinki and was approved by the ethics committee of Second Military Medical University. All participants provided written consent.

\section{Serum HBV genotyping and viral mutation analysis}

HBV DNA load, serological HBV markers, biochemical parameters for liver function, and platelet count were examined in the study hospitals. Details for viral DNA extraction, HBV genotyping, nested PCR, HBV sequencing (preS region, GenBank accession No. KF167178-KF169170), and viral mutation analysis are provided in the Supplementary Materials. The primers used for nested PCR are listed in Table S1. Twenty-six preS mutations were evaluated (Table S2), which were reported to be associated with HCC risk in our previous case-control studies.[9-13]

\section{Plasmid Construction}

From HBV-infected patients in this cohort study, we amplified the fragments of wild-type preS1/preS2/S gene (WT, nt.2848-nt.835) and preS1/preS2/S mutants carrying G2950A/G2951A/A2962G/C2964A (M1), C3116T/T31C (M2), and preS2 deletion (nt.15-nt.56, M3). The fragments of WT, M1, M2, and M3 were inserted into EcoR/ restriction sites of a $S B$ transposon vector, pKT2-FAH-Caggs-SB, that contained the cDNA of Fah gene.[19] For the production of lentiviruses expressing preS1/preS2/S variants, the fragments of WT, M1, M2, and M3 were linked with a flag tag at 3' end and were insirted into the plteni-CMV-GFP-puro lentiviral vector. The production of lentiviruses expressing preS1/preS2/S variants and the construction of luciferase reporter plasmid containing the promoter of signal transducer and activator of transcription 3 (STAT3) are detailed in the Supplementary Materials.

\section{Cell experiments}


Huh7 and HeLa cell lines were purchased from the Chinese Academy of Sciences (Shanghai, China). Before experiments, all cell lines were authenticated using the genotyping analysis of short tandem repeat (STR) by Biowing Biotechnology (Shanghai, China). All cell cultures were tested for mycoplasma contamination every three months. The cells stably expressing WT-preS1/preS2/S and preS1/preS2/S mutants were constructed with lentivirus. Interleukin-5 (IL-5) and IL-6 were purchased from R\&D Systems Inc. (Minneapolis, MN). STAT3 inhibitor Stattic and dimethylsulfoxide (DMSO) were purchased from Sigma (St. Louis, MO). Cell proliferation, migration, invasion, cell cycle assay, luciferase assay, real-time quantitative reverse transcription PCR (qRT-PCR), and Western blot are detailed in the Supplementary Materials. The primers used for qRT-PCR and the antibodies used for Western blot are listed in Tables S1 and S3, respectively. The levels of HBsAg in cells and culture medium were determined by ELISA (Jianglai, Shanghai, China). The intracellular HBsAg retention was presented as the ratio of intracellular HBsAg level to the HBsAg level in culture medium. The group of WT was applied as reference to calculate the change fold of intracellular HBsAg retention.

\section{Immunofluorescence staining}

Cells were grown on glass coverslips, fixed in 4\% paraformaldehyde, and permeabilized with $0.5 \%$ Triton X-100. Fixed cells were blocked with $5 \% \mathrm{BSA}$, incubated overnight at $4{ }^{\circ} \mathrm{C}$ with primary antibody, washed, incubated with secondary antibody, and counterstained with DAPI (Sigma). Primary and secondary antibodies were listed in Tables S3.

\section{Mouse models}

The Fah-deficient mouse was applied to construct the model of preS1/preS2/S-induced HCC.[19] The Fah ${ }^{-/}$mice were maintained with drinking water containing $7.5 \mu \mathrm{g} / \mathrm{mL}$ 2-(2-nitro-4-trifluoromethylbenzoyl)-1, 3-cyclohexanedione (NTBC). The constructs carrying Fah cDNA and the fragment of WT, M1, M2, or M3 (15 $\mu \mathrm{g})$ were delivered into 5-7week-old mice by hydrodynamic injection via tail vein. Then, NTBC was removed, and the mice that died within 30 days after injection were excluded from analysis due to the failure in gene delivery. The remaining mice were observed for six months to record survival, and they were all sacrificed on Day 180 after the injection. The livers and tumors were subjected to analyses of hematoxylin-eosin (H\&E) and immunohistochemistry (IHC). Serum samples were subjected to cytokine assessment, that was detailed in the Supplementary Materials. For the xenograft experiment, Nod-SCID mice (fiveweek-old, Jihui Laboratory Animal Care Cooperation, Shanghai, China) were subcutaneously injected with $1.5 \times 10^{6}$ cells. Tumors were harvested five weeks later. All animal studies were conducted under the animal welfare protocol approved by the ethics committee of Second Military Medical University.

\section{HBV-capture sequencing}

Six tissues of the M2-injected $S B$ mice were subjected to HBV-capture sequencing, including three liver tissues from tumor-free mice and three tumor tissues from tumor-carrying mice. HBV-capture sequencing was performed with the 
capture probes of the HBV panel (iGeneTech, Beijing, China) and the HiSeqTM 2500 platform (Illumina, San Diego, CA). Details for DNA extraction, DNA library construction, sequencing, and annotation of HBV integrated breakpoints are described in the Supplementary Materials. HBV-capture sequencing data were uploaded to the Sequence Read Archive (SRA) database under accession number of PRJNA765888.

\section{Gene expression profiling analysis}

The gene expression profiles of Huh7 cells were analyzed by RNA-sequencing (RNA-seq). Sequencing libraries were constructed using TruSeq Stranded Total RNA with Ribo-Zero Gold Kits (Illumina, San Diego, CA). The HiSeqTM 2500 sequencing platform (Illumina, San Diego, CA) was applied for RNA-seq. Twelve SB mice tissues were subjected to cDNA microarray analysis by using Agilent-074809 SurePrint G3 Mouse GE V2.0 microarray (Agilent Technologies, Santa Clara, CA), including three livers from WT-injected mice and three tumors from each group of preS1/preS2/S mutant-injected mice. Differential gene expression analysis, gene ontology (GO) analysis, and Kyoto Encyclopedia of Genes and Genomes (KEGG) pathway analysis are detailed in the Supplementary Materials. The gene profiling data of $S B$ mice and Huh7 cells were uploaded to the Gene Expression Omnibus database (accession number, GSE179125) and the SRA database (accession number, PRJNA762495).

\section{Statistical analysis}

The continuous variables, non-normal data, and categorical variables were compared using Student's t-test, Wilcoxon sum rank test, and Chi-square test, respectively. The Hazard ratio (HR) and 95\% confidence interval (Cl) were estimated using Cox regression analysis. The significant factors in the univariate Cox analysis were enrolled into the multivariate Cox model. For animal experiments, survival curves were compared by log-rank test. The analysis of the cohort data and experimental data was performed using SPSS (version 18.0, SPSS Inc., Chicago, IL) and GraphPad Prism (version 5.0, GraphPad Software, San Diego, CA), respectively. All statistical tests were two-sided. $P<0.05$ was considered statistically significant.

\section{Results}

\section{Effects of HBV preS mutations on the risk of HCC occurrence}

The 2114 patients were followed up for a median of 11.67 years (IQR, 7.58-15.17 years). Baseline characteristics and follow-up data are shown in Table S4. In total, 612 patients completed antiviral treatment for $\geq 48$ weeks. HBV genotype was successfully identified in 1659 patients. HBV genotype $\mathrm{C}$ and genotype B accounted for $57.30 \%$ and $21.10 \%$ of cases, respectively. During 23,845 person-year of follow-up, 224 HBV-infected patients developed HCC (incidence: 9.39/1000 person-years). 
In all HBV-infected patients, univariate Cox analyses demonstrated C3116T and T31C as significant risk factors for HCC occurrence, with HRs (95\% Cl) of 1.70 (1.20-2.40) and 1.60 (1.12-2.28), respectively. Male gender, age, cirrhosis, HBV genotype $\mathrm{C}$, high direct bilirubin, low albumin, high alpha-fetoprotein, and low platelet count were also identified as significant risk factors of $\mathrm{HCC}$, whereas antiviral treatment was a protective factor with an $\mathrm{HR}(95 \% \mathrm{Cl})$ of $0.54(0.38-$ 0.76). The preS deletion (pooling all deletion patterns together) was not statistically associated with HCC (data not shown). C3116T and T31C were not identified as independent risk factors by the multivariate Cox analysis (Table 1). Four HBV genotype C-specific mutations, G2950A, G2951A, A2962G, and C2964A, were identified as significant risk factors by the univariate Cox analyses in patients infected with genotype $\mathrm{C}$ HBV. Of these four single preS mutations, only A2962G was identified as an independent risk factor in the multivariate Cox model (Table S5).

Of genotype C HBV-infected patients, 2.18\% only presented one single mutation of G2950A, G2951A, A2962G, or C2964A, whereas $28.91 \%$ presented at least two of these four pres mutations, which were defined as combo mutation G2950A/G2951A/A2962G/C2964A positive (Figure 1A). Combo mutation G2950A/G2951A/A2962G/C2964A significantly increased the $\mathrm{HCC}$ risk in genotype $\mathrm{C}$ HBV-infected patients without antiviral treatment (age- and genderadjusted $\mathrm{HR}, 2.49 ; 95 \% \mathrm{Cl}, 1.08-5.72$ ), compared to those with antiviral treatment (Figure 1B). Of all HBV-infected patients, $17.43 \%$ presented only one single mutation of C3116 T or T31C, whereas $43.61 \%$ presented combo mutation C3116T/T31C (Figure 1C). C3116T/T31C significantly increased the HCC risk in patients without antiviral treatment (age- and gender-adjusted $\mathrm{HR}, 1.61 ; 95 \% \mathrm{Cl}, 1.06-2.45$ ) and was also associated with a trend toward increased $\mathrm{HCC}$ risk in patients who received antiviral treatment (Figure 1D). In preS gene, the region of nt.15-nt.56 showed the highest deletion frequency ( $\geq 7.16 \%$ ) and was defined as preS2 deletion (Figure 1E). Interestingly, preS2 deletion had no significant effect on the HCC risk of patients without antiviral treatment, but it significantly increased HCC risk in patients receiving antiviral treatment (age- and gender-adjusted $\mathrm{HR}, 4.11$; 95\% Cl, 1.57-10.72, Figure 1F).

\section{Effects of wild-type and mutant preS1/preS2/S on hepatocarcinogenesis and inflammation in SB mice}

The empty $S B$ vector and constructed plasmids were successfully delivered into $60 S B$ mice, 22 of which died during the six-month observation period. No control mice died. M3 was significantly associated with increased mortality of $S B$ mice (Figure 2A). In 38 surviving mice, no control mice developed tumor nodules in liver. Compared to WT-injected mice, all preS1/preS2/S mutant-injected mice displayed a trend toward a higher tumor burden (Figure 2B). The $S B$ system-induced integration sites were examined by HBV-capture sequencing in M2-injected mice, $57.14 \%$ of which developed tumor nodules. The preS1/preS2/S fragments were mainly integrated in intergenic region (39.36\%) and intron region (35.94\%). The number of integration sites was similar between tumor tissues and liver tissues (Figure 2C). Only three integrated genes were repeatedly detected in different samples, including microtubule associated serine/threonine kinase family member 4 (Mast4), transmembrane protein 178B (Tmem178b), and zinc finger BTB domain containing 20 (Zbtb20). These three genes all had a low ratio of integrated reads to normal reads (0.006$2.41 \%$, Table S6). Thus, tumor nodules in the $S B$ mice should not be caused by insertional mutations. The structure of cancer nest and extensive inflammatory cell infiltration were observed in the tumor nodule tissues from preS1/preS2/S mutant-injected mice (Figure 2D). The inflammatory pathological changes, including inflammatory cell infiltration, were more severe in the tissues of preS1/preS2/S mutant-injected mice than in those of WT-injected mice. The expression of HBV S protein, cytokeratin 18 (CK18, a classic HCC biomarker), and Ki67 (a biomarker for proliferation) was confirmed by IHC in tumors (Figure 2D). The serum levels of three T helper 1 (Th1) cytokines (IFNy, IL- $\beta 1$, and IL-12), one Th2 
cytokine (IL-5), and IL-6 were significantly upregulated in the preS1/preS2/S mutant-injected mice, compared with the WT-injected counterparts; however, this difference was not observed in the serum levels of tumor necrosis factor a (TNFa), transforming growth factor $\beta$ (TGF $\beta 1$ ), and vascular endothelial growth factor (VEGF) (Figure 3A). The serum level of IL-12 was significantly higher in the tumor-bearing mice than in the tumor-free mice; moreover, the tumorbearing mice also displayed a trend toward higher serum levels of IFNY, IL-5, and IL-6 (Figure 3B). No significant difference was observed in the serum levels of TNFa, IL1- $\beta 1$, TGF $\beta 1$, and VEGF between tumor-free mice and tumorbearing mice (Figure S1).

\section{Effects of preS1/preS2/S mutants on malignant phenotypes of cancer cells}

The oncogenic effects of preS1/preS2/S mutants were first investigated in an HCC cell line (Huh7). Compared to WT counterparts, stable ectopic expression of M2 and M3 significantly increased the proliferation and migration of Huh7 cells (Figure 4A,B). In HeLa cells, stable ectopic expression of three preS1/preS2/S mutants all significantly increased cell proliferation and only M3 showed a positive effect on cell migration (Figure 4C,D). None of the preS1/preS2/S mutants showed an obvious effect on cell cycle of Huh7 and HeLa cells (Figure S2). The in vivo experiments showed that ectopic expression of M3 significantly increased the weight of xenograft tumors of Huh7 and HeLa cells, compared to WT counterparts (Figure 4E). The ectopic expression of M1 and M2 in Huh7 also displayed a trend toward increasing the weight of xenograft tumors. Thus, the oncogenic effects of the preS mutations are not liver-specific.

\section{Bioinformatics analysis for the function of pres mutations}

Based on RNA-seq data of Huh7 cells and CDNA microarray data of tissues from $S B$ mice, we identified the differentially expressed genes (DEGs, $\mathrm{P}<0.05$, fold change $\geq 2$ ) in the groups of preS1/preS2/S mutants vs. the corresponding WT counterparts, respectively. More preS1/preS2/S mutant-induced DEGs $(n=1377)$ were identified in $S B$ mice than in Huh7 cells $(n=440)$ (Figure 5A). Among 937 DEGs specifically identified in mice, five oncogenes were randomly selected for qRT-PCR validation in Huh7 cells, including Wnt family member 4 (WNT4), ETS homologous factor $(E H F)$, phosphoglycerate dehydrogenase $(P H G D H)$, laminin subunit gamma 2 (LAMC2), and mucin 13 (MUC13). Interestingly, all of these oncogenes were upregulated by stimulation of IL-5 and/or IL-6 rather than the ectopic expression of preS1/preS2/S mutants (Table S7). Among these five oncogenes, WNT4 was significantly upregulated by both IL-5 and IL-6 rather than any preS1/preS2/S mutant (Figure 5B).[20] The preS1/preS2/S mutantinduced DEGs that were identified specifically in $S B$ mice (mice-DEGs) and those identified commonly in both $S B$ mice and Huh7 cells (common-DEGs) were subjected to functional analysis independently. By GO analysis, ten functional terms associated with inflammation and immune response were identified in mice-DEGs, whereas only two were identified in common-DEGs (Figure 5C). Among the other GO terms identified in this study, all of four STAT-related terms, three of four ER stress-related terms, and both hypoxia terms were only identified in common-DEGs rather than in mice-DEGs (Figure 5D). KEGG analysis found that most (9/11) of the pathways associated with metabolism significantly enriched in the common-DEGs, rather than in the mice-DEGs (Figure 5E).

\section{STAT3 participated in the preS1/preS2/S mutant-induced hepatocarcinogenesis}


Among STAT family members, STAT3 has been proved to be involved in the development of many cancers. Compared to WT, the ectopic expression of M2 and M3 significantly upregulated the protein level of STAT3 in both HeLa and Huh7 cells, whereas the ectopic expression of M1 only upregulated the protein level of STAT3 in Huh7 cells (Figure 6A). The protein levels of STAT3 and IL-6, the activator of STAT3 pathway, were significantly higher in the tissues adjacent to tumor from M2- and M3-injected mice, compared to their expression in the livers from WT-injected mice (Figure $6 \mathrm{~B}, \mathrm{C})$. However, the ectopic expression of preS1/preS2/S mutants had no effects on the mRNA level and the promoter activity of STAT3 (Figure S3). As mentioned above, the stable overexpression of M2 and M3 significantly promoted the proliferation of both HeLa and Huh7 cells. These effects were significantly attenuated by the the inhibitor of STAT3 pathway, Stattic. (Figure 6D).

\section{preS1/preS2/S mutants enhanced ER stress}

We then examined the expression levels of four downstream molecules of ER stress, namely endoplasmic reticulum oxidoreductase 1 alpha (ERO1L), protein kinase R-like ER kinase (PERK), C/EBP homologous protein (CHOP), and X-box binding protein-1 (XBP1).[21,22] The PERK/CHOP axis and XBP1 both control the cellular responses to ER stress via regulating transcription.[21] ERO1L promotes the disulfide bond formation of proteins. Compared to the WT, M2 and M3 significantly upregulated all four ER stress molecules in Huh7 cells, whereas M1 only upregulated ERO1L and XBP1 (Figure 7A). ER01L and PERK were further selected for the validation in tissues from SB mice. The protein levels of ERO1L and PERK were significantly higher in tumor tissues from M2 and preS2 deletion groups, compared to their expression in the liver tissues from WT-preS1/preS2/S-injected mice (Figure 7B,C). The ER stress-related pathological change, ground-glass hepatocytes (GGH) [23], was more obvious in liver tissues from each group of preS1/preS2/S mutant-injected $S B$ mice, compared to the WT-injected $S B$ mice (Figure 7C). The retention of HBsAg in ER was a major reason for ER stress. The effects of preS mutations on HBsAg retention were firstly evaluated by ELISA. In comparison with WT-expressing Huh7 cells, the ratio of intracellular HBsAg level to the HBsAg level in medium was significantly higher in M2- and M3-expressing Huh7 cells (Figure 7D). Immunofluorescence staining was further performed. In Huh7 cells expressing the WT, diffuse distribution of HBsAg and calnexin, a ER marker, was observed. However, the staining of HBsAg and calnexin showed a coarse and granular clustering pattern in the perinuclear area of cells expressing the preS1/preS2/S mutants. The colocalization of coarse dot-like cytoplasmic staining of HBsAg and calnexin was observed (Figure 7E). Thus, preS mutations, especially M2 and M3, may enhanced the retention of HBsAg in ER.

\section{Discussion}

In this study, we demonstrated that antiviral treatment not only was an independent protective factor of HCC in HBVinfected patients but also influenced the effects of preS mutations on the HCC risk. Combo mutations G2950A/G2951A/A2962G/C2964A and C3116T/T31C significantly increased the HCC risk only in HBV-infected patients without antiviral treatment. Interestingly, preS2 deletion significantly increased the risk of HCC in HBV-infected patients with antiviral treatment, indicating preS2 deletion may participate in drug resistance. Antiviral treatment was reported to be associated with increased frequency of preS deletion, especially in the preS2 region.[24] The preS deletion-induced GGH was an independent risk factor of HCC recurrence. Despite the reduction of serum HBV DNA levels, antiviral treatment with NA had no effect on the level of GGH.[25] These outcomes might partially be because the region of preS2 deletion overlaps the spacer domain of polymerase $(P)$ gene. The spacer region of HBV $P$ gene is a 
highly variable domain providing the flexibility for conformational changes and acts as a harbor for the treatmentselected HBV mutations.[26-28] The reverse transcriptase (rt) domain of HBV P gene overlaps with S gene. The rtA181T mutation, selected by lamivudine or adefovir, leads to sW172* mutation coding truncated HBsAg. The S mutant with sW172* induces ER stress, activates oncogenic pathways, and promotes hepatocarcinogenesis in cells and transgenic mice.[29] Similarly, pres2 deletion may cause viral polymerase mutations leading to drug resistance, which promotes HCC.

Compared to the WT, preS2 deletion induced a higher tumor burden in SB mice, increased cell proliferation in vitro, and promoted xenograft tumor growth. These results are consistent with previous studies demonstrating the oncogenic effect of preS deletion in cell models, HBV transgenic mouse models, and the models of HBV infection. $[9,23,30]$ For the first time, we found that G2950A/G2951A/A2962G/C2964A and C3116T/T31C combo mutations displayed a similar effect of pres2 deletion on carcinogenesis. Compared to the WT, M1 and M2 induced a trend toward a higher tumor burden in $S B$ mouse models and xenograft models of Huh7 cells. The recognition of specific receptor in liver induces HBV hepatotropism.[31] After being introduced, the viral mutations in HBx gene play a pro-carcinogenic role in nonhepatic cells, like HeLa.[32] In this study, M2 promoted the proliferation of both Huh7 and HeLa cells, suggesting the oncogenic effects of preS mutations are also not liver-specific. These combo mutations, especially C3116T/T31C, may act as pro-oncogenic molecules through a similar mechanism by which pres 2 deletion induces carcinogenesis.

We found that preS2 deletion and preS combo mutations promote hepatocarcinogenesis via deteriorating the inflammatory microenvironment. The serum levels of IL-5 and IL-6 were significantly upregulated in the preS1/preS2/S mutant-injected mice, compared to the WT-injected mice. Besides, preS1/preS2/S mutants induced dramatic alteration in the transcriptome of tissues from $S B$ mice, an animal models with an intact immune system. Much fewer DEGs were observed in the WT-expressing cells vs. the preS1/preS2/S mutant-expressing cells. Furthermore, some DEGs specifically identified in SB mice, such as WNT4, were only upregulated in Huh7 cells by the stimulation of IL-5 and/or IL-6, rather than by the ectopic expression of preS1/preS2/S mutants. Therefore, HBV preS mutations might regulate the transcription of oncogenes indirectly via altering the inflammatory microenvironment in humans with an intact immune system. The high-risk preS mutations accumulated during hepatocarcinogenesis can lead to immune escape by inducing the loss of B-cell epitopes or T-cell epitopes in HBV envelope proteins.[33] In HBV-infected patients with normal immunity, the deletion rate of the pres2 nt.15-nt.56 region, which codes the B-cell epitopes, is higher than that of other preS2 regions. In immune-suppressed kidney-transplant patients with HBV infection, the deletion rate of nt.15nt.56 is lower than that of other regions.[28] Interestingly, our data suggest that the inflammation-selected preS mutations could deteriorate the inflammatory microenvironment in turn. In a study of preS2 deletion transgenic mice, the mRNA levels of pro-inflammatory (TNFa, IL-6, IL-1a, and IL-1 $\beta$ ) and chemoattractant cytokines were significantly upregulated in the liver tissue. The increased proliferation of leukocytes is also reported.[30] The HCC patients with HBV preS2 deletion in the circulation display a higher number of regulatory $T$ cells (Tregs) and a higher expression level of immune checkpoint molecule programmed death ligand 1 (PD-L1) in tumor tissues than do patients without preS2 deletion.[34,35] Both increased Tregs and PD-L1 are associated with a poor prognosis of HCC.[36,37] Therefore, the feedback between inflammation and the preS mutations contributes simultaneously to the immune tolerance of mutant $\mathrm{HBV}$ antigens and the maintenance of pro-oncogenic inflammation.

This study also found that the preS mutations promote hepatocarcinogenesis via activating the STAT3 pathway and ER stress. The inhibitor of STAT3 pathway significantly attenuated the positive effects of preS1/preS2/S mutants on the proliferation of HeLa and Huh7 cells. M2 and M3 directly upregulated the protein levels of STAT3 rather than influencing the transcription of STAT3 gene. The effects of preS mutations on the protein level of STAT3 may be due to the aberrant ER function. ER stress is activated in response to an accumulation of unfolded or misfolded proteins, such as mutated HBV surface proteins, in the ER lumen. In this study, M2 and preS2 deletion significantly enhanced the 
retention of HBsAg in ER, compared to the WT. Sustained activation of ER stress endows malignant cells with greater tumorigenic, metastatic, and drug-resistant capacity.[21] The ER stress sensors, PERK and XBP1, were both reported to promote the survival capacity of cells by activating STAT3.[38,39] PERK was also activated during the process of epithelial-to-mesenchymal transition.[40] M2 and M3 significantly upregulated the protein level of PERK and XBP1, whereas M1 significantly upregulated XBP1. M1, M2, and M3 all significantly upregulated the protein level of another ER stress molecule, ERO1L, in cells and SB mice. The PERK/CHOP axis, ERO1L, and XBP1 promote the tolerance of cells to the hypoxia microenvironment and alter the metabolism function of mitochondria.[21,22,41-43] Consistent with the results of these previous studies, the GO term KEGG pathways associated with hypoxia and metabolism were significantly enriched in the preS mutation-induced DEGs identified commonly in SB mice and Huh7 cells. The preS mutation-activated ER stress may also promote pro-oncogenic inflammation. ERO1L and XBP1 can activate the IL-6 pathway and promote the progression of lung cancer and HCC, respectively.[44,45] Thus, the pres mutations promote hepatocarcinogenesis possibly via inducing pro-oncogenic inflammatory cytokine and signaling pathways associated with metabolism, the two major pathways related to ER stress.

The preS region of HBV harbors a complex spectrum of viral mutations. Compared to preS2 deletion, the oncogenic effect of base substitution in preS gene was underestimated and the underlying mechanism was not investigated. In this study, we for the first time, provide evidence supporting the oncogenic function of C3116T/T31C and G2950A/G2951A/A2962G/C2964A combo mutations. Remarkably, C3116T/T31C had a stronger capacity for upregulating all the four investigated molecules of ER stress in Huh7 cells, compared to preS2 deletion. The prevalence of C3116T/T31C (43.61\%) and G2950A/G2951A/A2962G/C2964A (26.91\%) was higher than that of preS2 deletion (7.16\%). Thus, these two combo mutations may have potential clinical implications for a wider population of HBVinfected patients.

To rule out the confounding effects of exogenous CMV promoter induced HBsAg overexpression, the WT group was applied as controls in cell experiments. Even though, our study has other limitations. First, we did not experimentally verify the effect of preS2 deletion on drug resistance. Second, the cDNA microarray analyses were conducted in the liver tissues from WT-preS1/preS2/S-injected mice and the liver tissues with tumors from preS1/preS2/S mutant-injected mice, because it is difficult to dissolve liver tissues from the tumor tissues of preS1/preS2/S mutant-injected mice.

Conclusively, HBV combo mutations C3116T/T31C and G2950A/G2951A/A2962G/C2964A significantly increased the risk of HCC in patients without antiviral treatment, whereas pres2 deletion (nt.15-nt.56) significantly increased the risk of HCC in patients who received antiviral treatment. The preS1/preS2/S mutants can promote hepatocarcinogenesis via inducing ER stress, altering metabolism, and pro-oncogenic STAT3 inflammatory pathways. The HBV-infected subjects with antiviral treatment should be monitored for the generation of preS2 deletion. HBV-infected patients with HCC-risk preS mutations could benefit from treatments targeting IL-6/STAT3 pathway and ER stress-related pathways.

\section{Abbreviations}

ER, endoplasmic reticulum; NA, nucleos(t)ide analog; IFNa, interferon a; SB, Sleeping Beauty; Fah, fumarylacetoacetate hydrolase; WT, wild-type preS1/preS2/S gene; M1, preS1/preS2/S mutants carrying G2950A/G2951A/A2962G/C2964A; M2, C3116T/T31C; M3, preS2 deletion; STAT3, signal transducer and activator of transcription 3; STR, short tandem repeat; qRT-PCR, real-time quantitative reverse transcription PCR; NTBC, 2-(2-nitro-4-trifluoromethylbenzoyl)-1, 3cyclohexanedione; H\&E, hematoxylin-eosin; IHC, immunohistochemistry; SRA, Sequence Read Archive; RNA-seq, RNAsequencing; GO, gene ontology; KEGG, Kyoto Encyclopedia of Genes and Genomes; Mast4, microtubule associated serine/threonine kinase family member 4; Tmem178b, transmembrane protein 178B ; Zbtb20, zinc finger BTB domain containing 20; CK-18, cytokeratin 18; Th1, T helper 1; DEGs, the differentially expressed genes; WNT4, Wnt family member 4; EHF, ETS homologous factor; PHGDH, phosphoglycerate dehydrogenase; LAMC2, laminin subunit gamma 2;

Page $10 / 23$ 
MUC13, mucin 13; ERO1L, endoplasmic reticulum oxidoreductase 1 alpha; PERK, protein kinase R-like ER kinase; CHOP, C/EBP homologous protein; XBP1, X-box binding protein-1; HHG, ground-glass hepatocytes; PD-L1, programmed death ligand 1; GEO, Gene Expression Omnibus.

\section{Declarations}

Data Availability: The cDNA microarray data of $S B$ mouse models were uploaded to the Gene Expression Omnibus (GEO) database under accession number of GSE179125. The HBV-capture sequencing data of SB mice and the RNAsequencing data of Huh7 cells were uploaded to the Sequence Read Archive (SRA) database under accession numbers of PRJNA765888 and PRJNA762495, respectively.

Ethics, consent and permissions: The study protocol conformed to the 1975 Declaration of Helsinki and was approved by the ethics committee of Second Military Medical University. All participants provided written consent. All animal studies were conducted under the animal welfare protocol.

Financial support: This work was supported by grant 2015CB554006 from the National Key Basic Research Program of China (GC); grants 91529305 (GC), 81520108021 (GC), 81673250 (GC), 81521091 (GC), 82003538 (WL), and 81502882 (XC) from the National Natural Science Foundation of China; and grants GWV-10.1-XK17 from the "3-year public health promotion" program of Shanghai Municipal Health Commission (GC).

Competing interests: The authors declare that they have no competing interests.

Authors' contributions: Cao GW conceived and supervised the study. Pu R, Cai SL, Liu DH, Li ZX, Zhou XY, Hou XM, Chen LP, Wu JF, and Yang F carried out the experiments. Cai SL, Liu WB, and Chen X did the bioinformatics analysis. Liu WB and Cai SL conducted the statistical analyses and drafted the manuscript. Cao GW interpreted the data, discussed the results, and extensively revised the manuscript. Wang X provided the Sleeping Beauty mouse model. Tan XJ and Yin JH guided the experiments. All authors read and approved the final manuscript.

\section{References}

1. Sung H, Ferlay J, Siegel RL, Laversanne M, Soerjomataram I, Jemal A, et al. Global Cancer Statistics 2020: GLOBOCAN Estimates of Incidence and Mortality Worldwide for 36 Cancers in 185 Countries. CA Cancer J Clin. 2021;71(3):209-249.

2. Sagnelli E, Macera M, Russo A, Coppola N, Sagnelli C. Epidemiological and etiological variations in hepatocellular carcinoma. Infection. 2020;48(1):7-17.

3. Yang F, Ma L, Yang Y, Liu W, Zhao J, Chen X, et al. Contribution of Hepatitis B Virus Infection to the Aggressiveness of Primary Liver Cancer: A Clinical Epidemiological Study in Eastern China. Front Oncol. 2019;9:370. 
4. Yin J, Chen X, Li N, Han X, Liu W, Pu R, et al. Compartmentalized evolution of hepatitis B virus contributes differently to the prognosis of hepatocellular carcinoma. Carcinogenesis. 2021;42(3):461-470.

5. Cao GW. Cancer Evo-Dev, a novel hypothesis derived from studies on hepatitis B virus-induced carcinogenesis. Hepatoma Res. 2017;3:241-259.

6. Liu W, Wu J, Yang F, Ma L, Ni C, Hou X, et al. Genetic Polymorphisms Predisposing the Interleukin 6-Induced APOBEC3B-UNG Imbalance Increase HCC Risk via Promoting the Generation of APOBEC-Signature HBV Mutations. Clin Cancer Res. 2019;25(18):5525-5536.

7. Yin J, Zhang H, He Y, Xie J, Liu S, Chang W, et al. Distribution and hepatocellular carcinoma-related viral properties of hepatitis B virus genotypes in Mainland China: a community-based study. Cancer Epidemiol Biomarkers Prev. 2010;19(3):777-786.

8. Xie JX, Zhao J, Yin JH, Zhang Q, Pu R, Lu WY, et al. Association of novel mutations and haplotypes in the preS region of hepatitis B virus with hepatocellular carcinoma. Front Med China. 2010;4(4):419-429.

9. Yin J, Xie J, Zhang H, Shen Q, Han L, Lu W, et al. Significant association of different preS mutations with hepatitis Brelated cirrhosis or hepatocellular carcinoma. J Gastroenterol. 2010;45(10):1063-1071.

10. Chen $\mathrm{CH}$, Hung $\mathrm{CH}$, Lee $\mathrm{CM}, \mathrm{Hu} \mathrm{TH}$, Wang JH, Wang JC, et al. Pre-S deletion and complex mutations of hepatitis $B$ virus related to advanced liver disease in HBeAg-negative patients. Gastroenterology. 2007;133(5):1466-74.

11. Taltavull TC, Sata T, Abe K. High prevalence of hepatitis B virus pre-s mutant in countries where it is endemic and its relationship with genotype and chronicity. J Clin Microbiol. 2003;41(12):5449-55.

12. Chen $X$, Zhang M, Li N, Pu R, Wu T, Ding Y, et al. Nucleotide variants in hepatitis B virus preS region predict the recurrence of hepatocellular carcinoma. Aging (Albany NY). 2021;13(18):22256-22275.

13. Lin WL, Hung JH, Huang W. Association of the Hepatitis B Virus Large Surface Protein with Viral Infectivity and Endoplasmic Reticulum Stress-mediated Liver Carcinogenesis. Cells. 2020;9(9):2052.

14. Hung JH, Su IJ, Lei HY, Wang HC, Lin WC, Chang WT, et al. Endoplasmic reticulum stress stimulates the expression of cyclooxygenase-2 through activation of NF-kappaB and pp38 mitogen-activated protein kinase. J Biol Chem. 2004;279(45):46384-46392.

15. Liu H, Xu J, Zhou L, Yun X, Chen L, Wang S, et al. Hepatitis B virus large surface antigen promotes liver carcinogenesis by activating the Src/PI3K/Akt pathway. Cancer Res. 2011;71(24):7547-7557.

16. Liang YJ, Teng W, Chen CL, Sun CP, Teng RD, Huang YH, et al. Clinical Implications of HBV PreS/S Mutations and the Effects of PreS2 Deletion on Mitochondria, Liver Fibrosis, and Cancer Development. Hepatology. 2021;74(2):641655.

17. Yin J, Li N, Han Y, Xue J, Deng Y, Shi J, et al. Effect of antiviral treatment with nucleotide/nucleoside analogs on postoperative prognosis of hepatitis B virus-related hepatocellular carcinoma: a two-stage longitudinal clinical study. $J$ Clin Oncol. 2013;31(29):3647-3655.

18. Yin J, Wang J, Pu R, Xin H, Li Z, Han X, et al. Hepatitis B Virus Combo Mutations Improve the Prediction and Active Prophylaxis of Hepatocellular Carcinoma: A Clinic-Based Cohort Study. Cancer Prev Res (Phila). 2015;8(10):978988. 
19. Wangensteen KJ, Wilber A, Keng VW, He Z, Matise I, Wangensteen L, et al. A facile method for somatic, lifelong manipulation of multiple genes in the mouse liver. Hepatology. 2008;47(5):1714-1724.

20. Yang D, Li Q, Shang R, Yao L, Wu L, Zhang M, et al. WNT4 secreted by tumor tissues promotes tumor progression in colorectal cancer by activation of the Wnt/ $\beta$-catenin signalling pathway. J Exp Clin Cancer Res. 2020;39(1):251.

21. Cubillos-Ruiz JR, Bettigole SE, Glimcher LH. Tumorigenic and Immunosuppressive Effects of Endoplasmic Reticulum Stress in Cancer. Cell. 2017;168(4):692-706.

22. Zhang J, Yang J, Lin C, Liu W, Huo Y, Yang M, et al. Endoplasmic Reticulum stress-dependent expression of ERO1L promotes aerobic glycolysis in Pancreatic Cancer. Theranostics. 2020;10(18):8400-8414.

23. Yang JC, Teng CF, Wu HC, Tsai HW, Chuang HC, Tsai TF, et al. Enhanced expression of vascular endothelial growth factor-A in ground glass hepatocytes and its implication in hepatitis B virus hepatocarcinogenesis.

Hepatology. 2009;49(6):1962-71.

24. Zhang D, Dong P, Zhang K, Deng L, Bach C, Chen W, et al. Whole genome HBV deletion profiles and the accumulation of preS deletion mutant during antiviral treatment. BMC Microbiol. 2012;12:307.

25. Tsai HW, Lin YJ, Wu HC, Chang TT, Wu IC, Cheng PN, et al. Resistance of ground glass hepatocytes to oral antivirals in chronic hepatitis B patients and implication for the development of hepatocellular carcinoma.

Oncotarget. 2016;7(19):27724-27734.

26. Chen P, Gan Y, Han N, Fang W, Li J, Zhao F, et al. Computational evolutionary analysis of the overlapped surface (S) and polymerase $(\mathrm{P})$ region in hepatitis $\mathrm{B}$ virus indicates the spacer domain in $\mathrm{P}$ is crucial for survival. PLoS One. 2013;8(4):e60098.

27. Campo DS, Dimitrova Z, Lara J, Purdy M, Thai H, Ramachandran S, et al. Coordinated evolution of the hepatitis B virus polymerase. In Silico Biol. 2012;11(5-6):175-182.

28. Yin F, Xie Y, Fan H, Zhang J, Guo Z. Mutations in hepatitis B virus polymerase are associated with the postoperative survival of hepatocellular carcinoma patients. PLoS One. 2017;12(12):e0189730.

29. Lai MW, Liang KH, Lin WR, Huang YH, Huang SF, Chen TC, et al. Hepatocarcinogenesis in transgenic mice carrying hepatitis B virus pre-S/S gene with the sW172* mutation. Oncogenesis. 2016;5(12):e273.

30. Teng YC, Neo JC, Wu JC, Chen YF, Kao CH, Tsai TF. Expression of a hepatitis B virus pre-S2 deletion mutant in the liver results in hepatomegaly and hepatocellular carcinoma in mice. J Pathol. 2017;241(4):463-474.

31. Schieck A, Schulze A, Gähler C, Müller T, Haberkorn U, Alexandrov A, et al. Hepatitis B virus hepatotropism is mediated by specific receptor recognition in the liver and not restricted to susceptible hosts. Hepatology. 2013;58:4353.

32. Chae S, Ji JH, Kwon SH, Lee HS, Lim JM, Kang D, et al. HBxAPa/Rsf-1-mediated HBx-hBubR1 interactions regulate the mitotic spindle checkpoint and chromosome instability. Carcinogenesis. 2013;34(7):1680-8.

33. Sun H, Chang L, Yan Y, Wang L. Hepatitis B virus pre-S region: Clinical implications and applications. Rev Med Virol. 2020. doi: 10.1002/rmv.2201. 
34. Teng CF, Li TC, Wang T, Liao DC, Wen YH, Wu TH, et al. Increased infiltration of regulatory T cells in hepatocellular carcinoma of patients with hepatitis B virus pre-S2 mutant. Sci Rep. 2021;11(1):1136.

35. Teng CF, Li TC, Wang T, Wu TH, Wang J, Wu HC, et al. Increased Expression of Programmed Death Ligand 1 in Hepatocellular Carcinoma of Patients with Hepatitis B Virus Pre-S2 Mutant. J Hepatocell Carcinoma. 2020;7:385-401.

36. Fu J, Xu D, Liu Z, Shi M, Zhao P, Fu B, et al. Increased regulatory T cells correlate with CD8 T-cell impairment and poor survival in hepatocellular carcinoma patients. Gastroenterology. 2007;132(7):2328-2339.

37. Gao Q, Wang XY, Qiu SJ, Yamato I, Sho M, Nakajima Y, et al. Overexpression of PD-L1 significantly associates with tumor aggressiveness and postoperative recurrence in human hepatocellular carcinoma. Clin Cancer Res. 2009;15(3):971-979.

38. Aydin Y, Kurt R, Song K, Lin D, Osman H, Youngquist B, et al. Hepatic Stress Response in HCV Infection Promotes STAT3-Mediated Inhibition of HNF4A-miR-122 Feedback Loop in Liver Fibrosis and Cancer Progression. Cancers (Basel). 2019;11(10):1407.

39. Gonnella R, Gilardini Montani MS, Guttieri L, Romeo MA, Santarelli R, Cirone M. IRE1 Alpha/XBP1 Axis Sustains Primary Effusion Lymphoma Cell Survival by Promoting Cytokine Release and STAT3 Activation. Biomedicines. 2021;9(2):118.

40. Feng YX, Sokol ES, Del Vecchio CA, Sanduja S, Claessen JH, Proia TA, et al. Epithelial-to-mesenchymal transition activates PERK-elF2a and sensitizes cells to endoplasmic reticulum stress. Cancer Discov. 2014;4(6):702-15.

41. Gonen N, Meller A, Sabath N, Shalgi R. Amino Acid Biosynthesis Regulation during Endoplasmic Reticulum Stress Is Coupled to Protein Expression Demands. iScience. 2019;19:204-213.

42. Chen X, lliopoulos D, Zhang Q, Tang Q, Greenblatt MB, Hatziapostolou M, et al. XBP1 promotes triple-negative breast cancer by controlling the HIF1a pathway. Nature. 2014;508(7494):103-107.

43. Mujcic H, Nagelkerke A, Rouschop KM, Chung S, Chaudary N, Span PN, et al. Hypoxic activation of the PERK/elF2a arm of the unfolded protein response promotes metastasis through induction of LAMP3. Clin Cancer Res. 2013;19(22):6126-37.

44. Lei Y, Zang R, Lu Z, Zhang G, Huang J, Liu C, et al. ER01L promotes IL6/sIL6R signaling and regulates MUC16 expression to promote CA125 secretion and the metastasis of lung cancer cells. Cell Death Dis. 2020;11(10):853.

45. Fang P, Xiang L, Huang S, Jin L, Zhou G, Zhuge L, et al. IRE1a-XBP1 signaling pathway regulates IL-6 expression and promotes progression of hepatocellular carcinoma. Oncol Lett. 2018;16(4):4729-4736.

\section{Table}

Table 1. Univariate and multivariate Cox regression analysis of factors significantly affected the occurrence of hepatocellular carcinoma 


\begin{tabular}{|c|c|c|c|c|c|c|c|}
\hline Variable & $\begin{array}{l}\text { No. }(\%) \text { of } \\
\text { participants } \\
(n=2114)\end{array}$ & $\begin{array}{l}\text { Person- } \\
\text { years } \\
\text { of } \\
\text { follow- } \\
\text { up }\end{array}$ & $\begin{array}{l}\text { No. of } \\
\mathrm{HCC} \\
(n=224)\end{array}$ & $\begin{array}{l}\text { Incidence } \\
\text { rate per } \\
1000 \\
\text { person- } \\
\text { years }\end{array}$ & $\begin{array}{l}\text { Univariate } \\
\text { analysis } \\
\mathrm{HR}(95 \% \\
\mathrm{Cl})\end{array}$ & $P$ Value & $\begin{array}{l}\text { Multivariate } \\
\text { analysis HR } \\
(95 \% \mathrm{Cl})\end{array}$ \\
\hline
\end{tabular}

Gender

$\begin{array}{lllllllll}\text { Female } & 490(23.18) & 5987 & 30 & 5.01 & 1 & & 1 & \\ \text { Male } & 1624(76.82) & 17858 & 194 & 10.86 & \begin{array}{l}2.08(1.41- \\ 3.05)\end{array} & <0.001 & \begin{array}{l}3.28(1.78- \\ 6.04)\end{array} & <0.001\end{array}$

Age (years)

$\begin{array}{lllllllll}<60 & 1885(89.17) & 21363 & 190 & 8.89 & 1 & & 1 & \\ \geq 60 & 229(10.83) & 2482 & 34 & 13.70 & \begin{array}{l}1.62(1.12- \\ 2.33)\end{array} & 0.010 & \begin{array}{l}1.92(1.15- \\ 3.20)\end{array} & 0.013\end{array}$

Antiviral treatment

$\begin{array}{lllllllll}\text { No } & 1502(71.05) & 17361 & 184 & 10.60 & 1 & & 1 & \\ \text { Yes } & 612(28.95) & 6484 & 40 & 6.17 & \begin{array}{l}0.54(0.38- \\ 0.76)\end{array} & <0.001 & \begin{array}{l}0.61(0.39- \\ 0.96)\end{array} & 0.031\end{array}$

Cirrhosis

$\begin{array}{lllllllll}\text { No } & 1624(76.82) & 19372 & 126 & 6.50 & 1 & & 1 & \\ \text { Yes } & 490(23.18) & 4473 & 98 & 21.91 & \begin{array}{l}3.39(2.60- \\ 4.41)\end{array} & <0.001 & \begin{array}{l}2.04(1.25- \\ 3.32)\end{array} & 0.004\end{array}$

C3116T

$\begin{array}{lllllll}\mathrm{C} & 755(56.26) & 8403 & 56 & 6.66 & 1 & \\ \mathrm{~T} & 587(43.74) & 6765 & 75 & 11.09 & \begin{array}{l}1.70(1.20- \\ 2.40)\end{array} & 0.003\end{array}$

T31C

$\begin{array}{lllllll}\mathrm{T} & 675(52.61) & 7605 & 51 & 6.71 & 1 & \\ \mathrm{C} & 608(47.39) & 6898 & 73 & 10.58 & \begin{array}{l}1.60(1.12- \\ 2.28)\end{array} & 0.011\end{array}$

HBV genotype

$\begin{array}{lllllll}\text { B } & 447(26.94) & 4977 & 27 & 5.42 & 1 & \\ \text { C } & 1212(73.06) & 13887 & 137 & 9.87 & \begin{array}{l}1.86(1.23- \\ 2.81)\end{array} & 0.003\end{array}$

Direct bilirubin (mol/L)

$\begin{array}{lllllll}\leq 7 & 691(33.11) & 8370 & 56 & 6.69 & 1 & \\ >7 & 1396(66.89) & 15157 & 167 & 11.02 & \begin{array}{l}1.63(1.21- \\ 2.21)\end{array} & <0.001\end{array}$

Albumin (g/L)

$\begin{array}{llllll}\geq 35 & 1335(63.36) & 16255 & 113 & 6.95 & 1\end{array}$




$\begin{array}{lllllll}<35 & 772(36.64) & 7507 & 109 & 14.52 & \begin{array}{l}2.08(1.59- \\ 2.70)\end{array}\end{array}$

a-fetoprotein $(\mathrm{ng} / \mathrm{mL}$ )

$\begin{array}{lllllll}\leq 20 & 1358(64.36) & 16014 & 124 & 7.74 & 1 & \\ 20-400 & 752(35.64) & 7791 & 100 & 12.84 & \begin{array}{l}1.61(1.23- \\ 2.09)\end{array} & <0.001\end{array}$

Platelet count $\left(10^{9} / \mathrm{L}\right)$

\begin{tabular}{lllllll}
$100-300$ & $1004(47.49)$ & 12050 & 68 & 5.64 & 1 & \\
\hline$<100$ & $1110(52.51)$ & 11795 & 156 & 13.23 & $\begin{array}{l}2.35(1.77- \\
3.12)\end{array}$ & $<0.001$
\end{tabular}

\section{Figures}





D
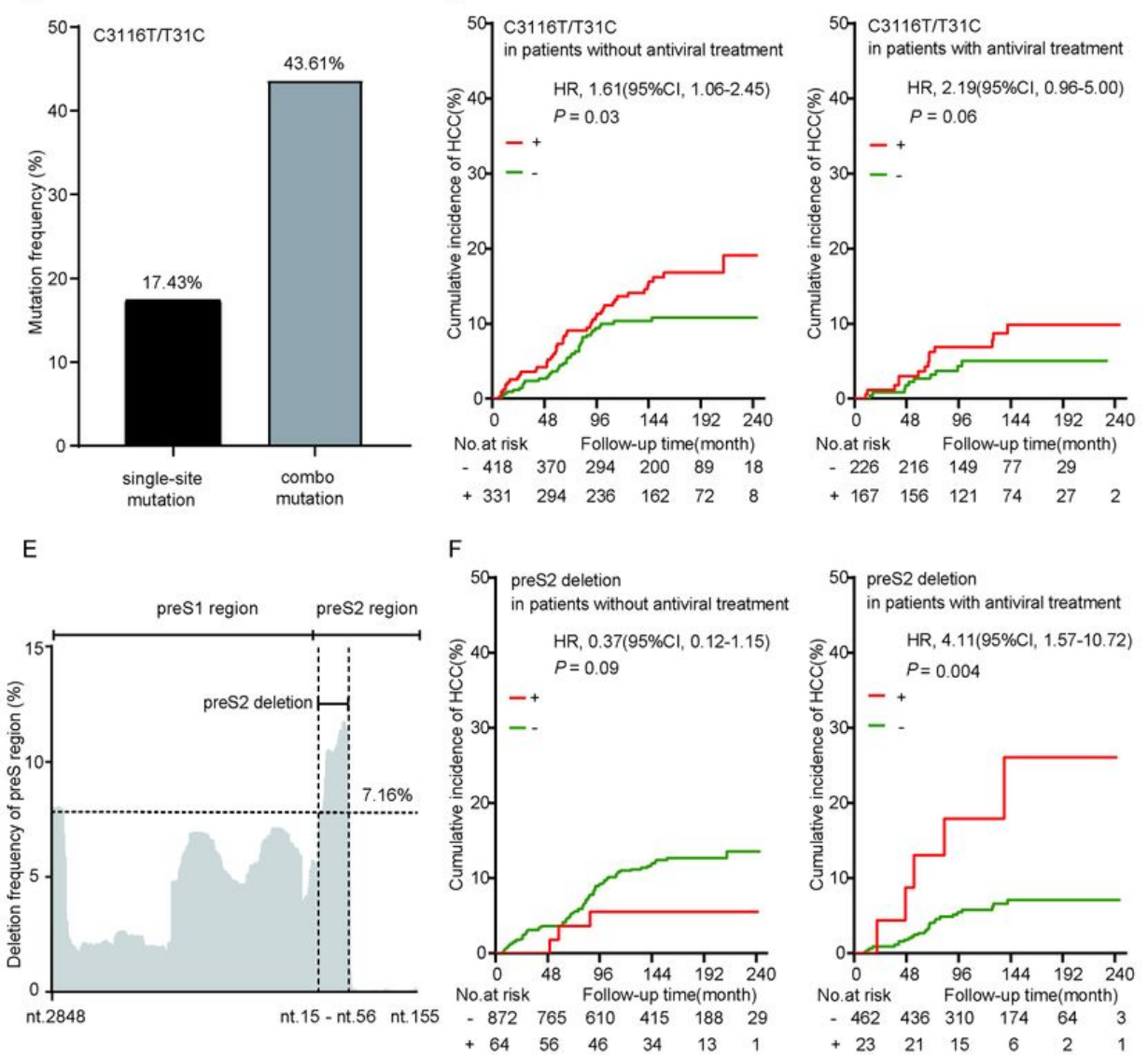

\section{Figure 1}

The impact of baseline preS mutations on HCC occurrence in a prospective cohort study with 2114 HBV-infected patients. (A) The frequencies of G2950A, G2951A, A2962G, and C2964A single mutations and G2950A/G2951A/A2962G/C2964A combo mutation. Patients with at least two of these four mutations were defined as combo mutation positive. (B) The effect of G2950A/G2951A/A2962G/C2964A combo mutation on HCC occurrence in genotype C HBV-infected patients without antiviral treatment (Left) and in those who received antiviral treatment (Right). (C) The frequencies of C3116T and T31C single mutations and C3116T/T31C combo mutation. (D) The effect of HCC C3116T/T31C combo mutation on HCC occurrence in all HBV-infected patients without antiviral treatment (Left) and in those who received antiviral treatment (Right). (E) The deletion frequency of each locus in the preS1/preS2 region. The region with the highest deletion rate (nt.15-nt.56) was defined as preS2 deletion in this study. (F) The effect 
of preS2 deletion on HCC occurrence in HBV-infected patients without antiviral treatment (Left) and in those who received antiviral treatment (Right).
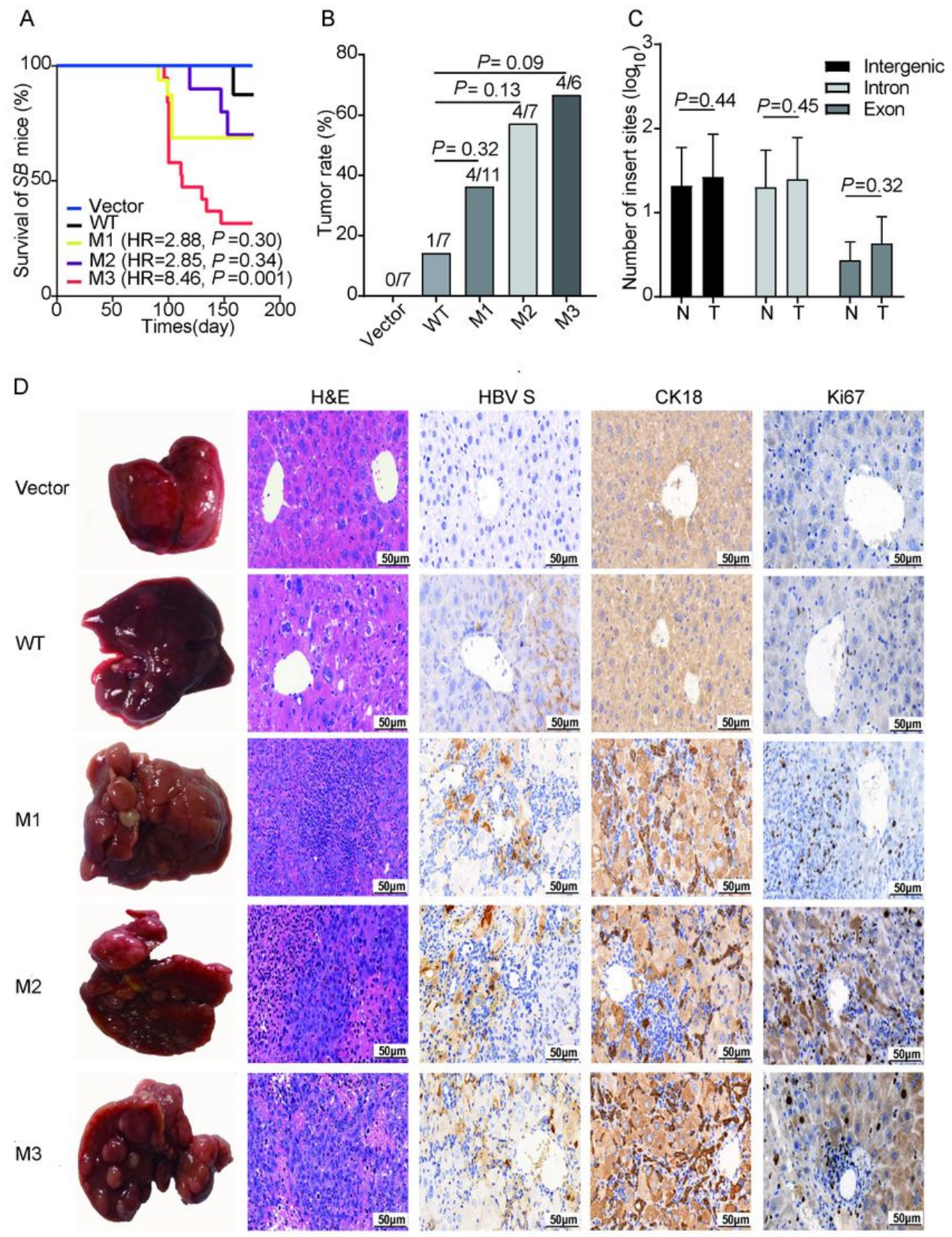

\section{Figure 2}

The survival, tumor occurence, HBV DNA insertion sites, and pathological characteristics of the Sleeping Beauty (SB) mouse models. (A) The survival of $S B$ mice during six-month observation. Vector, WT, M1, M2, and M3 indicate the mouse models injected with the empty vector, wild-type preS1/preS2/S, G2950A/G2951A/A2962G/C2964A, C3116T/T31C, and preS2 deletion, respectively. (B) The rate of tumor occurrence in SB mouse models. (C) The number of HBV preS1/preS2/S insertion sites in different functional regions and different tissue types. T, tumors from the mice with tumor nodules; N, liver tissues from the tumor-free mice. (D) Representative images of H\&E and IHC staining of the livers (from Vector and WT groups) and tumors (from M1, M2, and M3 groups). Column 1, representative gross 
features; Column 2, representative H\&E staining; Columns 3-5, representative IHC staining for HBV S protein, CK-18, and Ki67, respectively.
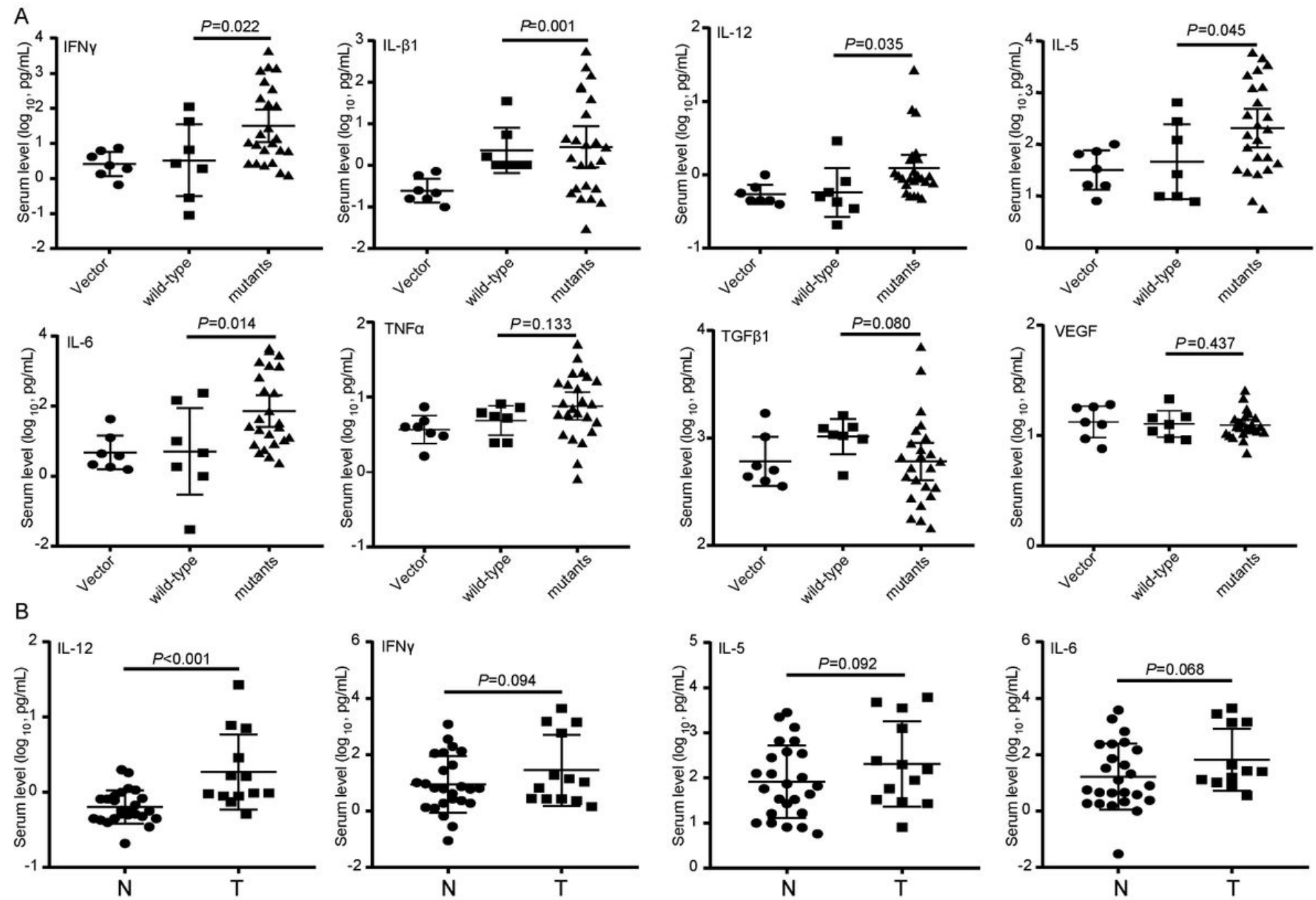

Figure 3

The serum levels of cytokines in SB mouse models. (A) Serum levels of IFNy, IL- $\beta 1$, IL-12, IL-5, IL-6, TNFa, TGF $\beta 1$, and VEGF. Vector, the empty vector-injected mice; wild-type, WT-injected mice; mutants, preS1/preS2/S mutant-injected mice. (B) Serum levels of IL-12, IFNy, IL-5, and IL-6 in the mouse models with or without tumor. N, the tumor-free mice; T, the mice with tumors. 

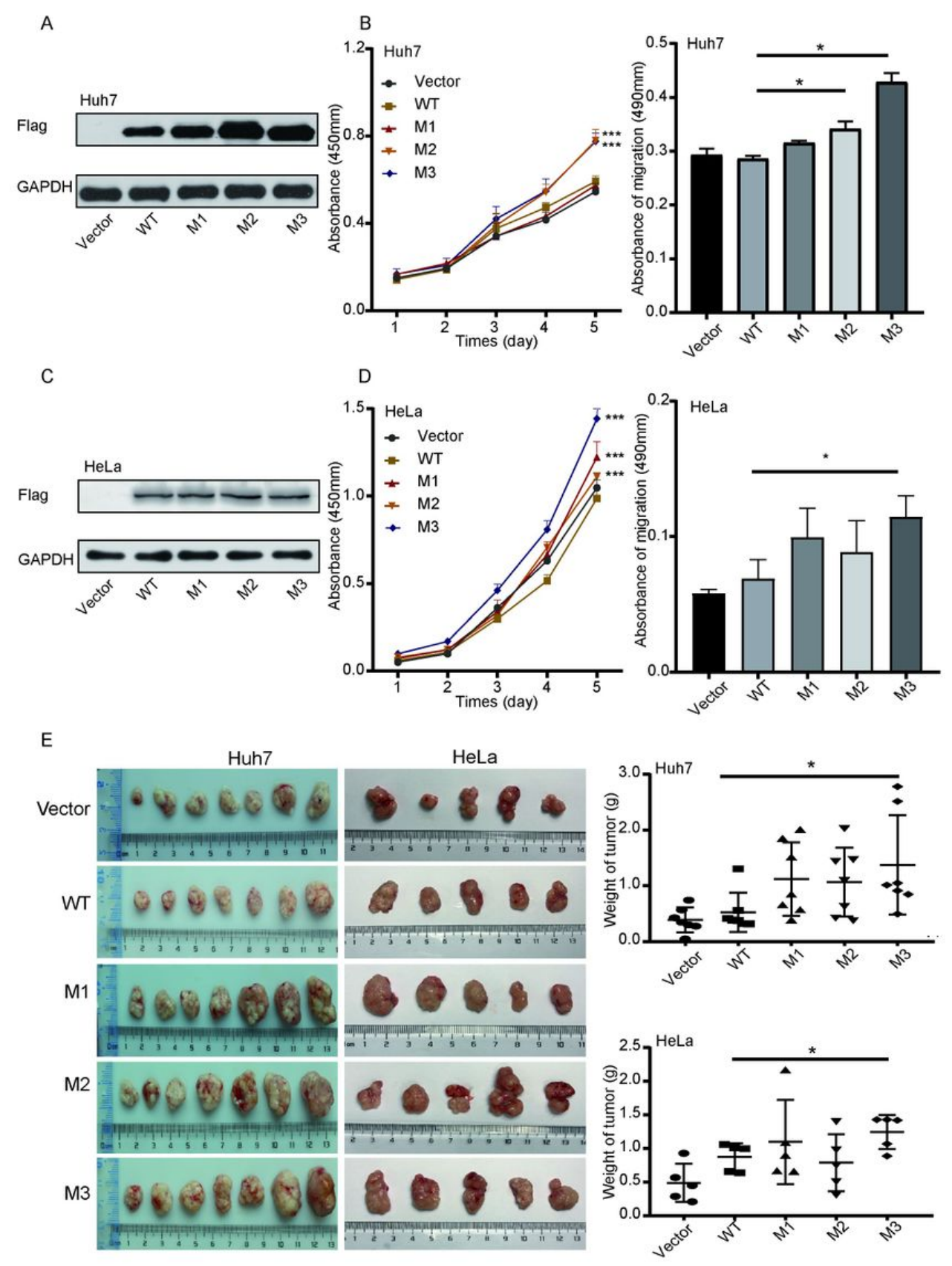

Figure 4

Effects of preS1/preS2/S mutations on malignant phenotypes of cancer cells. (A) Ectropic overexpression of HBV preS1/preS2/S mutants and WT counterpart was confirmed by detecting Flag tag in Huh7 cells. (B) Effects of the preS1/preS2/S mutants on the proliferation (Left) and migration (Right) of Huh7 cells. (C) Ectropic overexpression of HBV preS1/preS2/S mutants and WT counterpart was confirmed by detecting Flag tag in HeLa cells. (D) Effects of the preS1/preS2/S mutants on the proliferation (Left) and migration (Right) of HeLa cells. (E) The weight of xenograft tumors among different groups. $* P<0.05, * \star * P<0.001$. 
A

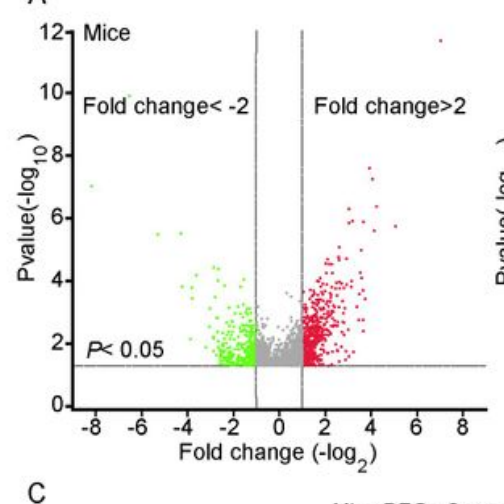

C

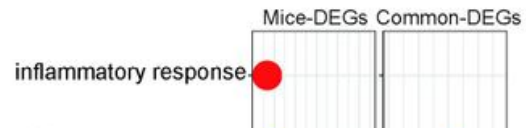

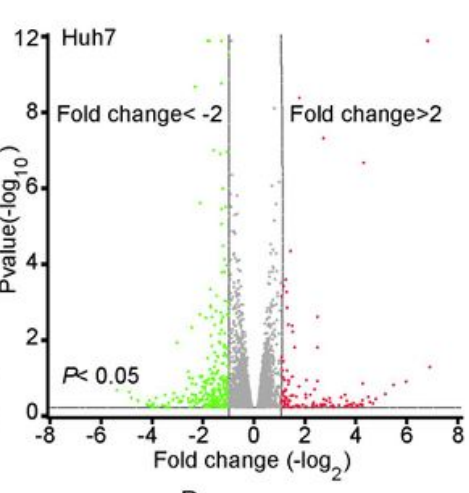

D
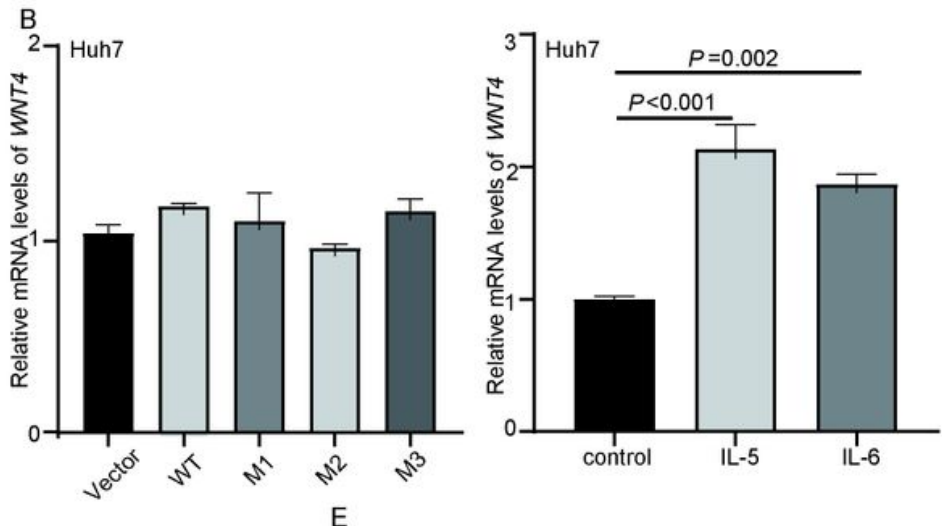

Mice-DEGs Common-DEGs

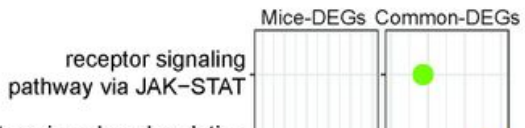

Biosynthesis of amino acids

Micer

\section{Figure 5}

Functional analysis of HBV preS mutations. (A) (Left) Differential expressed genes (DEGs) in preS1/preS2/S mutantinjected $S B$ mice vs. WT preS1/preS2/S-injected $S B$ mice $(\mathrm{n}=1377)$. (Right) DEGs in HBV preS1/preS2/S mutantexpressing Huh7 cells vs. HBV preS1/preS2/S WT-expressing Huh7 cells $(n=440)$. (B) The mRNA levels of WNT4 in Huh7 cells which is detected by qRT-PCR. (Left) Ectopic expression of HBV preS1/preS2/S mutants has no direct effect on the mRNA expression of WNT4. (Right) Effects of IL-5 and IL-6 (100 ng/mL, $1 \mathrm{~h})$, two preS1/preS2/S mutantinduced cytokines, on the mRNA expression of WNT4. (C) Most of the inflammation- and immune response-related GO terms were significantly enriched in DEGs that were identified specifically in $S B$ mice (mice-DEGs) rather than in DEGs identified both in $S B$ mice and Huh7 cells (common-DEGs). (D) ER stress-, STAT pathway-, and hypoxia-related GO terms were significantly enriched in common-DEGs, rather than in the mice-DEGs. (E) Most of the KEGG identified metabolism-related pathways significantly enriched in common-DEGs, rather than in the mice-DEGs. 

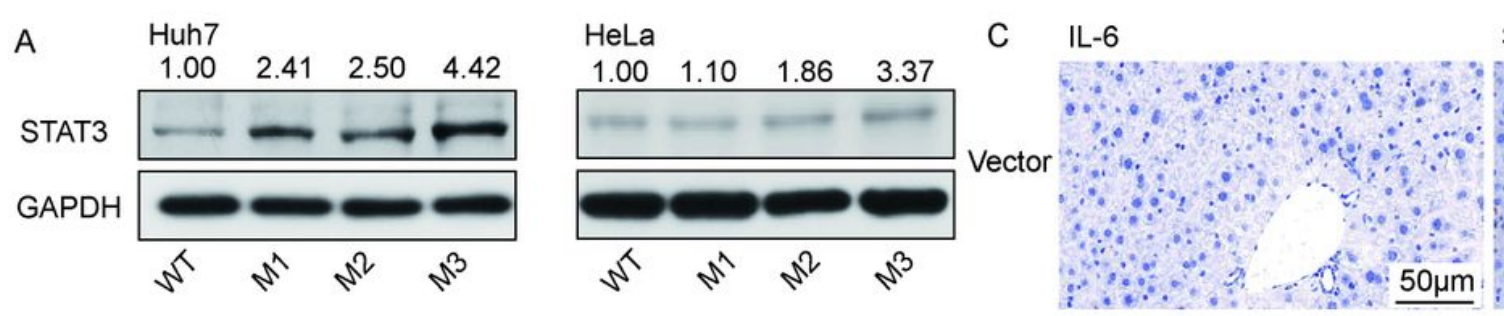

STAT3
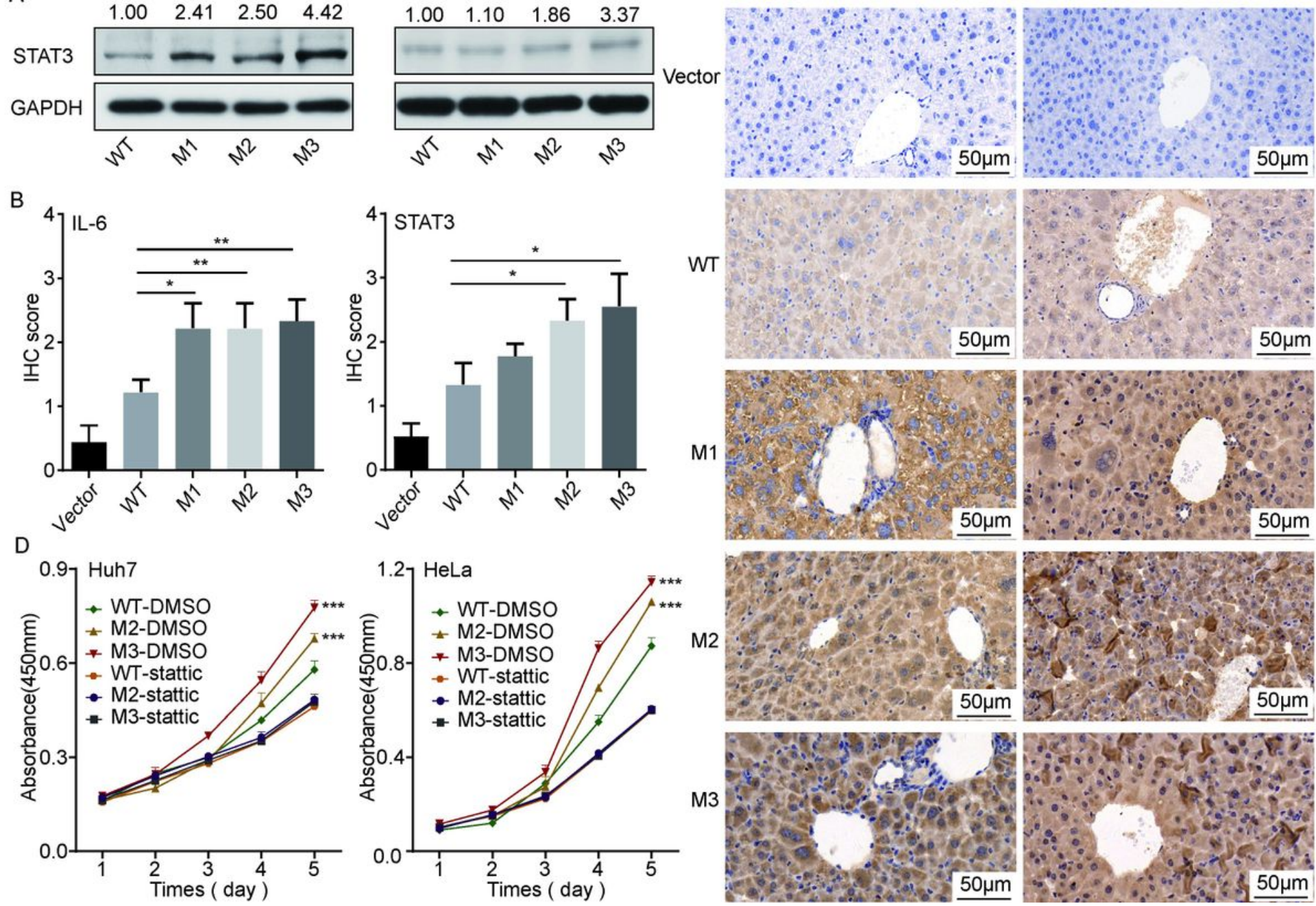

\section{Figure 6}

The roles of STAT3 in the preS1/preS2/S mutant-induced hepatocarcinogenesis. (A) The protein level of STAT3 was higher in Huh7 and HeLa cells with ectopic expression of preS1/preS2/S mutants than in those with ectopic expression of the WT counterpart. (B) The histograms displaying the IHC scores of IL-6 and STAT3 in the liver tissues (vector group and WT group) and tissues adjacent to tumors (groups of preS1/preS2/S mutants) from SB mice. (C) Representative images for the IHC staining of IL-6 and STAT3 in adjacent liver tissues to tumors. (D) The effects of mutant HBV S proteins on the cell proliferation in Huh7 and HeLa cells. In cells treated with $0.1 \%$ DMSO, M2 and M3 significantly increased the proliferation of HeLa and Huh7 cells, compared with WT. In cells treated with $10 \mu \mathrm{M}$ Stattic, no significant difference was observed in the proliferation among cell expressing M2, M3, and WT.

\section{Figure 7}

The preS1/preS2/S mutants significantly enhanced ER stress and the retention of HBsAg in ER. (A) The protein levels of ER01L, PERK, CHOP, and XBP1 in Huh7 cells with the ectopic expression of WT or preS1/preS2/S mutants. (B) Representative IHC and H\&E images. Column 1 and 2 show the IHC staining of EROL 1 and PERK. In column 3, the ER stress-related pathological change, ground-glass hepatocytes, was more obvious in liver tissues from each group of preS1/preS2/S mutant-injected SB mice. (C) The histograms displaying the IHC scores of EROL1 and PERK in the liver 
tissues (vector group and WT group) and tumor tissues (groups of preS1/preS2/S mutants) from SB mice. (D) The change fold of intracellular HBsAg retention. The level of HBsAg in cells and medium was evaluated by ELISA. The HBsAg was presented by the ratio of intracellular HBsAg level to the HBsAg level in medium. The WT group was applied as reference to calculate change fold. $(E)$ The result of immunofluorescence staining. Huh7 cells with the stable expression of WT, M1, M2, or M3 were immunostained with mouse anti-HBsAg (green) and rabbit anti-calnexin antibodies (red), with DAPI (blue) as nuclear counterstain. $\star \star \star ~ P<0.001$.

\section{Supplementary Files}

This is a list of supplementary files associated with this preprint. Click to download.

- FigureS1.pdf

- Figures2.pdf

- Figures3.pdf

- Supplementorymaterial.doc

- Tables1.docx

- Tables2.docx

- Tables3.docx

- Tables4.docx

- Tables5.docx

- Tables6.docx

- Tables7.docx 